\title{
The effects of costless pre-play communication: Experimental evidence from games with Pareto-ranked equilibria
}

\author{
Andreas Blume ${ }^{\mathrm{a}}$, Andreas Ortmann ${ }^{\mathrm{b}, *}$ \\ ${ }^{a}$ Economics Department, University of Pittsburgh, Pittsburgh, PA 15260, USA \\ ${ }^{\mathrm{b}}$ Center for Economic Research and Graduate Education, Charles University, Economics Institute, \\ Academy of Sciences of the Czech Republic, PO Box 882, Politickych veznu, 7, CZ 11121 Prague, \\ Czech Republic
}

Received 16 July 2000; final version received 3 March 2005

Available online 13 June 2005

\begin{abstract}
Cheap talk is shown to facilitate coordination on the unique efficient equilibrium in experimental order-statistic games. This result is roughly consistent with theoretical predictions according to which cheap talk promotes efficient Nash play. The evidence concerning the mechanisms that theory appeals to is mixed: Frequent agreement of messages and actions is consistent with messages being viewed as self-committing. Risk in the underlying game and the absence of self-signaling messages may explain why message profiles are not unanimous. Time-varying message profiles can be interpreted as evidence for players trying to negotiate equilibria and/or trying to rely on secret handshakes.

(C) 2005 Elsevier Inc. All rights reserved.
\end{abstract}

JEL classification: C72; C92

Keywords: Coordination games; Order-statistic games; Cheap talk; Experiments

\section{Introduction}

We experimentally investigate the effects of costless pre-play communication, or cheap talk, in symmetric coordination games of the stag hunt variety [11-15]. Van Huyck, Battalio, and Beil (VHBB) [33,34] demonstrate that in so-called Minimum and Median games with

\footnotetext{
* Corresponding author. Fax: +420224227143.

E-mail address: andreas.ortmann@cerge-ei.cz (A. Ortmann).
} 
multiple Pareto-ranked equilibria, the Pareto-efficient equilibria need not be selected, and in fact typically are not selected [7, Chapter 7.4]. Taking as the point of departure a Minimum game explored in VHBB [33], Berninghaus and Ehrhart [3] demonstrate the crucial importance of the frequency of play for these results. Building on a Median game design in VHBB [34], Van Huyck et al. [36] show that a dramatically refined action space allows cohorts of size 7 to creep toward efficiency in some cases. These two papers suggest that the consequence of the reduced opportunity cost of experimentation that is the by-product of the increased number of plays in the first case and the refined action space in the second case can facilitate, but not guarantee, "tacit coordination".

VHBB [35] show that adding a pre-play auction each round enables experimental participants to coordinate on the Pareto-efficient equilibrium. Crawford and Broseta [16] provide a model of the efficiency-enhancing effects of the pre-play auctions in VHBB [35]. They point out that these auctions are a costly form of pre-play communication, or signaling, that draws on the power of forward induction. Building also on the Median game results in VHBB [34], Cachon and Camerer [6] show that asking participants to pay a fixed price for participation also enables participants to coordinate on the Pareto-efficient equilibrium. The VHBB [35] result, and the Crawford and Broseta [16] explanation of this result, suggest that the price of the right to play serves as another means of "tacit communication" that allows participants to eliminate equilibria with payoffs lower than that price, and thus to reduce the strategic uncertainty resulting from the multiplicity of equilibria. The CachonCamerer results suggest a role for both forward induction and loss avoidance. Devetag [18] establishes, for critical mass games that are closely related to the games under consideration here, the importance of the degree of information feedback.

In the experiments summarized above the signal is both tacit and costly. Costless signaling in coordination games has been investigated by Cooper, De Jong, Forsythe, and Ross (CDFR) [10]. They focus on two-player games with two Pareto-ranked equilibria and study one-sided as well as two-sided pre-play communication. They find that cheap talk has the potential to increase both the incidence of equilibrium play and the frequency of the efficient equilibrium being reached. For a two-by-two game with tension between Pareto efficiency and risk dominance, they find that one-sided communication somewhat increases the frequency of the efficient equilibrium being reached whereas two-sided communication guarantees efficiency. ${ }^{1}$ The results in VHBB [33], however, suggest that the results of two-player games may be very different from those that involve more than two players, although much of this seems to be driven by the matching protocol used (e.g., [33,9]).

It is well known that the addition of a cheap-talk stage to a game does not eliminate any equilibrium outcomes of the original game. After all, the cheap-talk announcements can simply be ignored. This argument does not accord well with our intuition for these games. Farrell [20] suggests a rationale for cheap talk's apparent effects. According to him, if the players' pre-play announcements constitute a Nash equilibrium, this equilibrium becomes a focal point that entices players to follow their announced plans. Subsequent theoretical

\footnotetext{
${ }^{1}$ Charness [8], Clark et al. [9] and Duffy and Feltovich [19] have recently re-examined similar games with somewhat different designs. Charness finds a stronger, and Clark et al. a weaker efficiency-enhancing role for pre-play communication. Duffy and Feltovich show that the effectiveness of pre-play communication is a function of the game. All agree that communication affects play.
} 
work, extensively discussed in Section 3 below, has provided additional arguments for the role of cheap talk (e.g., evolutionary ones), but also noted possible countervailing forces such as risk in the underlying game or the absence of self-signaling messages, i.e. messages the sender wants to send if and only if they are true (see [1,21]).

In the following we investigate these theoretical considerations by combining the experimental frameworks of VHBB [33,34] and CDFR [10]. In particular, we ask whether costless pre-play communication with a priori meaningful messages by all players is effective in coordination games with more than two players.

The paper is organized as follows: Section 2 describes the experimental design and implementation of our experimental sessions, while Section 3 reviews the theory for games with pre-play communication that is relevant for our experiments. In Section 4 we report the results of the experimental sessions and discuss them in light of the theory. Section 5 concludes.

\section{Experimental design and implementation}

Following the advice of Davis and Holt [17, p. 520] not to change too many things at once, we employed the key earnings tables in VHBB [34,33] to facilitate comparison with previous experimental results. We also replicated their results in "baseline treatments" to establish that our results in the pre-play treatments were not driven by experimenter effects, subject pool effects, or other experimental artifacts.

The coordination games represented by the two earnings tables in Table 1 (ETMe) and Table 2 (ETMin), involve two or more players who choose among seven actions, or efforts, with the labeling being typically such that the higher effort (which in equilibrium leads to higher payoffs) is labeled with the higher number. This labeling establishes the a priori meaning of the action labels relevant for some of the theoretical considerations. The generating functions underlying the earnings tables are similar albeit not the same. ${ }^{2}$ Specifically, earnings losses resulting from deviations from the relevant order statistic are nonlinear, and increasing (asymmetrically) in the degree of deviation, for the Median game, while they are linear for the Minimum game. ${ }^{3}$ Importantly, both types of coordination games have the same set of seven symmetric, Pareto-ranked, pure strategy equilibria on the main diagonal. Clearly, the highest Pareto-ranked equilibrium is located in the upper left corner in both payoff tables and requires each player's highest effort.

\footnotetext{
2 The generating function for ETMe is $\pi\left(e_{i}, \mathrm{Me}\right)=a e_{i}-b\left(\left(e_{i}-\mathrm{Me}+1\right)^{2}-1\right)+c$, with $a=\$ .1, b=\$ .05$, and $c=\$ .6$; Me denotes the median of all choices and $e_{i}$ denotes player $i$ 's choice, with $i=1, \ldots, 9$. The generating function for ETMin is $\pi\left(e_{i}, e_{-i}\right)=a\left(\operatorname{Min}\left(e_{i}, e_{-i}\right)\right)-b e_{i}+c$, with $a=\$ .2, b=\$ .1, c=\$ .6 ; e_{-i}$ denotes all actions except that of the $i$ th player, again with $i=1, \ldots, 9$.

3 The coordination games represented in ETMe and ETMin derive their names, "Median game", and "Minimum game", respectively, from the order statistics employed rather than the specific parameterization. The fact that both the order statistic and the specific parameterization were different in VHBB [33,34] is a potential confound. Clearly, an alternative route to go would be to apply both order statistics investigated here to one or the other parameterization. That is, we could have made ETMe into a Minimum game and ETMin into a Median game. For reasons of better comparability, we followed the precedent set in the literature. Doing so is arguably a tougher test of the effects of pre-play communication, as it is the higher penalties for deviations in the earnings table underlying the Median case that produced the initial distribution of actions. More on this later.
} 
Table 1

Earnings table for Median game (ETMe)

\begin{tabular}{rllllllrr}
\hline \multicolumn{10}{c}{ Median value of $X$ chosen } \\
\hline Your choice of $X$ & 7 & 1.30 & 6 & 5 & 4 & 3 & \multicolumn{1}{c}{2} & 1 \\
& 6 & 1.25 & 1.20 & 1.05 & 0.80 & 0.45 & 0.00 & -0.55 \\
& 5 & 1.10 & 1.15 & 1.10 & 0.95 & 0.70 & 0.35 & -0.10 \\
& 4 & 0.85 & 1.00 & 1.05 & 1.00 & 0.85 & 0.60 & 0.25 \\
& 3 & 0.50 & 0.75 & 0.90 & 0.95 & 0.90 & 0.75 & 0.50 \\
& 2 & 0.05 & 0.40 & 0.65 & 0.80 & 0.85 & 0.80 & 0.65 \\
& 1 & -0.50 & -0.05 & 0.30 & 0.55 & 0.70 & 0.75 & 0.70 \\
\hline
\end{tabular}

Table 2

Earnings table for Minimum game (ETMin)

\begin{tabular}{|c|c|c|c|c|c|c|c|c|}
\hline & & \multicolumn{7}{|c|}{ Smallest value of $X$ chosen } \\
\hline & & 7 & 6 & 5 & 4 & 3 & 2 & 1 \\
\hline \multirow{7}{*}{ Your choice of $X$} & 7 & 1.30 & 1.10 & 0.90 & 0.70 & 0.50 & 0.30 & 0.10 \\
\hline & 6 & & 1.20 & 1.00 & 0.80 & 0.60 & 0.40 & 0.20 \\
\hline & 5 & & & 1.10 & 0.90 & 0.70 & 0.50 & 0.30 \\
\hline & 4 & & & & 1.00 & 0.80 & 0.60 & 0.40 \\
\hline & 3 & & & & & 0.90 & 0.70 & 0.50 \\
\hline & 2 & & & & & & 0.80 & 0.60 \\
\hline & 1 & & & & & & & 0.70 \\
\hline
\end{tabular}

Table 3

Design matrix: treatments

\begin{tabular}{lll}
\hline & Baseline (w/o) & Pre-play (w/) \\
\hline Median (Me) sessions & 8 & 8 \\
Minimum (Min) sessions & B1Me-B8Me & M1Me-M8Me \\
& 4 & 8 \\
& B1Min-B4Min & M1Min-M8Min \\
\hline
\end{tabular}

A total of 28 sessions were conducted that were distributed across the four treatments as detailed in Table 3.

Baseline sessions, labeled B1Me through B8Me, and B1Min through B4Min, consisted of 8 action stages each. ${ }^{4}$ Pre-play sessions, labeled M1Me through M8Me, and M1Min through M8Min, consisted of 8 action stages each and a message stage preceding each of the action stages. ${ }^{5}$ In a message stage, every participant entered the choice "he or she wishes

\footnotetext{
${ }^{4}$ The imbalance in the number of baseline sessions was a deliberate choice. Minimum game results without pre-play communication have been documented to be extremely robust. There was ex ante, and there is ex post, no good reason to run more sessions. In contrast, Median game results are less robust (e.g., [34,6]).

${ }^{5}$ Median sessions employed either earnings table ETMe or ETMe*; both earnings tables are identical except that ETMe has the Pareto-dominant equilibrium in the upper left corner while ETMe* has it in the lower right. ETMe* was employed to counteract the possibility of the game design becoming known on campus, a possibility that is small but real at a residential college (see implementation discussion below).
} 
to send." The instructions continued as follows: "Messages you may choose are 1, 2, 3, 4, $5,6,7$. You may choose a message to indicate the value (action) that you plan to choose in the action stage." No explicit mapping between messages and actions was provided but clearly the meaning of a message was suggestive. The distribution of messages was communicated to participants via screen display. ${ }^{6}$ Subsequently subjects were prompted for their action choices as in the baseline treatment. Subjects received information about their payoffs and the realized value of the order statistic but received no other information about the distribution of actions. They were informed about payoffs after each round.

Following VHBB [34], but somewhat deviating from VHBB [33] ${ }^{7}$, we employed nine participants per session, for a total of $9 \times 28=252$ subjects. These subjects were asked repeatedly to make choices among the seven actions that were available to them. For each round, participants' payoffs were determined by their own choices and the relevant order statistic of all subjects' choices in their session. Instructions, adopted from VHBB [34,33], made participants familiar with the experimental setting and made it common information. A questionnaire ensured that participants knew how to read the earnings table and how to compute a median.

All Median sessions were conducted at Bowdoin College, a selective residential liberal arts college in Maine whose population is more homogeneous (age, race) than the average US college. All Minimum sessions were conducted at the University of Pittsburgh. ${ }^{8}$ In both cases subjects were recruited via e-mail. Once they arrived they were seated. The experimenter then read the instructions aloud.

All sessions were conducted in a computerized environment that made record keeping in principle unnecessary. However, we asked participants to keep record sheets and to record their own choices, the median or minimum choices, and their per-round and total earnings. The eight rounds reported here took-including the instructions-about half an hour per session.

\section{Theoretical considerations}

In this section, we briefly review the theory for signaling intentions in cheap-talk games and relate it to our experimental design in order to prepare for the discussion of experimental results in the following section.

\footnotetext{
${ }^{6}$ The communication was non-graphical and simply stated, for $i=1, \ldots, 7$, "Number of players who sent message $i$ : _." Selected screenshots, including the one illustrating this communication screen, are available from the corresponding author and at http://home.cerge-ei.cz/ortmann/instructions.html

${ }^{7}$ VHBB [33] employ larger groups. Since strategic uncertainty is positively related to group size (as VHBB [33] show), we chose the same number of participants in both games to reduce the number of possible confounds.

${ }^{8}$ The location choices were forced on us. We ran the Median experiments first at Bowdoin College where the second author was then teaching. When, prompted by an associate editor and a referee for this journal, we decided to run the Minimum experiments, the second author had already moved on to an institution in Europe and running a new set of experiments at Bowdoin College was not an option. We hence decided to run the Minimum experiments at the University of Pittsburgh. While there is the possibility that subject pool and experimenter effects influence results, our baseline results, which replicate the qualitative results from earlier experiments, suggest strongly that such effects play a negligible role if any.
} 
As Luce and Raiffa [27, p. 172] point out, “... the equilibrium notion does not serve in general as a guide to action." Therefore Nash equilibria are frequently viewed as selfenforcing agreements (e.g. [29, p. 106] or [26, p. 411]). One envisions that players meet before the game to engage in pre-play communication. For any agreement emerging from such a meeting to be stable, it will have to be a Nash equilibrium. Furthermore, some have expressed the belief that agents will negotiate efficient agreements. This suggests two central conjectures concerning the role of pre-play communication: (1) Pre-play communication promotes Nash equilibrium play. (2) Pre-play communication promotes Pareto-efficient Nash equilibrium play.

In our games, with multiple Pareto-ranked equilibria, the first conjecture in isolation can be viewed as suggesting that pre-play communication reduces the variance of play in the first round. The second conjecture suggests that in either game we expect play to be concentrated near the unique efficient equilibrium.

Theoretical support for both of these conjectures, however, is mixed. Consider a communication game consisting of a finite simultaneous-move game, the base game (e.g., our Median or Minimum game), preceded by a number of rounds of communication. This mere conversion of the base game into a communication game does not establish either of the two conjectured roles for communication. Instead of strategic uncertainty in the base game, players now face strategic uncertainty in the communication game. Communication does not automatically lead to equilibrium selection, as all equilibria of the base game can be recovered as equilibria in the communication game in which communication is simply ignored.

Some have made the argument that communication should be effective when there are credible messages (see e.g., [21]). This line of reasoning invokes the existence of a natural language whose meanings are understood and hence seems applicable to our experimental design (recall that messages in our design have a natural association with actions). Consider the case where just one player communicates. Then one possible standard for the credibility of a declaration of intent is that it be self-committing: The sender must have an incentive to conform with his declaration if he expects it to be believed. Thus, a message announcing to play a strategy that is part of a strict Nash equilibrium would be credible. Moreover, the talking player can induce his preferred Nash equilibrium. In this case communication would induce an efficient Nash equilibrium, confirming both of the above conjectures.

This positive conclusion can be challenged on several grounds, which we will argue below are relevant to the games we consider. One famous challenge by Aumann [1] seeks to strengthen the credibility standard. It poses that for a message to be credible, it has to be self-signaling: The sender wants it to be believed if and only if he is truthful. In other words, it should not be possible for a receiver of a message to respond to the sender, "You would have said that anyway." In a game in which the sender's preferences over his opponent's actions are independent of his own planned action choice, no self-signaling message exists. Hence, according to this argument, in such a game there is no role for communication to promote Nash equilibria, let alone efficient ones.

A second challenge (see [4]) points out the role of risk in the underlying game. Communication may reduce but not entirely remove strategic uncertainty in the base game. Then the force of self-commitment seems diminished if there is a safer alternative strategy. The riskier an equilibrium is in the base game, the heavier is the burden on communication 
to reassure the listener of the speaker's intent and to reassure the speaker himself of the effectiveness of his communication. ${ }^{9}$

A third challenge might note the role of the communication protocol, i.e. the number of players who are communicating, the order in which they talk and the frequency with which they talk. Although the self-commitment and self-signaling notions retain an intuitive appeal, the definitions for the case with one player communicating do not directly apply to alternative communication protocols. With multiple players talking (as in our design), the credibility of a message has to be judged in the context of other messages. For example, with a large number of players sending simultaneous messages, a player's proposal to play a particular Nash equilibrium is hardly credible if all other players agree on an alternative equilibrium. Perhaps one should try to define credibility for message profiles, rather than for individual messages. It is not even clear, however, what interpretation, if any, to give to a heterogeneous message profile. While a unanimous profile may have meaning and be credible, both the meaning and credibility of a heterogeneous message profile may be in doubt.

Multiple communication rounds preceding the base game may provide the opportunity to repeatedly try to achieve unanimity and thus to negotiate Nash equilibria as in Farrell [20], Rabin [30] and Jamison [24]. On the other hand, if communication alternates with play (as in our design), i.e., the communication game is repeated, there is the possibility that message meanings degrade. One cannot be sure that a priori meanings remain stable after histories in which actions have not conformed with messages.

On the positive side, repetition of the communication game may provide opportunities for learning and for abandoning unsuccessful message profiles: According to the secret handshake argument (e.g., [31]) that is frequently invoked in the evolutionary literature on pre-play communication in games (e.g., $[28,37,25,23,4]$ ), players can costlessly adjust their play after unsent messages and start sending those messages when it becomes advantageous. In a large class of games this leads to selection of efficient Nash equilibria, which would be in line with both of the conjectures stated above. Kim and Sobel [25] consider a simple dynamic process of strategy adjustment, and show that in two-player common interest games there is a unique stable outcome, which is efficient. Hurkens [23] shows for any finite number of players that if the underlying game has a strict equilibrium that gives all communicating players their highest payoffs, and if messages are distinguished by negligibly small message costs, then the communication game has a unique curb (closed under rational behavior) retract [2] and all strategies in this retract support the efficient equilibrium. Blume [4] obtains a similar result without message costs by assuming that messages have some a priori information content (AIC). AIC formalizes the idea that if all players communicate and their messages express unanimity about an equilibrium, then this equilibrium becomes slightly more attractive for the communicating players.

Our design lets us look at a number of these issues. The Minimum game appears to be more sensitive to strategic uncertainty than the Median game. The highest action (ranked in terms of payoffs of the corresponding strict Nash equilibria) carries more risk in the Minimum game than in the Median game by a variety of measures. For example, in the

\footnotetext{
${ }^{9}$ Burton and Sefton [5] experimentally investigate the role of risk in communication games with a unique equilibrium in the base game.
} 
Minimum game a deviation of a single player from the efficient Nash equilibrium causes all players to wish they had deviated themselves, whereas in the Median game a single deviation from the efficient Nash equilibrium leaves the median unaltered. The maximin action in the Minimum game corresponds to the strict Nash equilibrium with the lowest payoff, whereas in the Median game it corresponds to the third lowest payoff. Increasing heterogeneity in the action profile lowers the minimum action without necessarily affecting the median. Thus, if we think of the variance of action profiles as a measure of strategic uncertainty, increasing strategic uncertainty tends to promote use of the minimal action in the Minimum game whereas in the Median game there is no such effect.

Leaving aside the issue of how to precisely define self-signaling messages with multiple communicating players, there is a sense in which messages are self-signaling in the Median game, and not in the Minimum game. In the Minimum game, each player (weakly) prefers that the other players raise their actions. In particular, regardless of his intended play, say action 4, a player is willing to send message 7, if he believes that this message will induce all other players to take action 7. In contrast, in the Median game, a player intent on using action 4 is willing to send message 5 but not message 7 , if he believes his message to decide the median action. In this sense, we will say that there are self-signaling messages in the Median game, and not in the Minimum game.

Despite the fact that there are self-signaling messages in the Median game, it does not follow that we should expect the unique efficient Nash equilibrium to be played in the one-shot version of the communication game. The reason is the strategic uncertainty in the communication game and the difficulty of interpreting heterogeneous message profiles. A player who expects that a message profile will induce a median below the maximum may well wish to send a message that reinforces that belief rather than to increase uncertainty by sending a message that would signal his intent to take the maximal action. ${ }^{10}$

Finally, repetition of the communication game permits us to look for evidence of secret handshakes that would promote efficient equilibrium selection or degradation of meanings, which might have a negative effect on communication outcomes.

\section{Experimental results}

In this section, we report our experimental results and use them to reflect on the theory and vice versa. We begin by describing aggregate behavior for the four treatments across time. We then take a more detailed look at first-round play, terminal play, the dynamics of actions, message-action associations, and the dynamics of messages.

Figs. 1 and 2 compare choices in all baseline and pre-play Median and Minimum sessions, respectively. For each treatment, the figures display the average and median choice of actions (messages) in each round aggregated across sessions.

The results in the left panels of Figs. 1 and 2 are fully in line with results reported in the literature (e.g., [34,6,33]). Across all baseline sessions of the Median game the median action was 5 in the first round (with more than 60 percent of the action choices being either

\footnotetext{
${ }^{10}$ This is reminiscent of the observation that players may use messages to secure a payoff rather than to promote efficiency (e.g., [5]).
} 

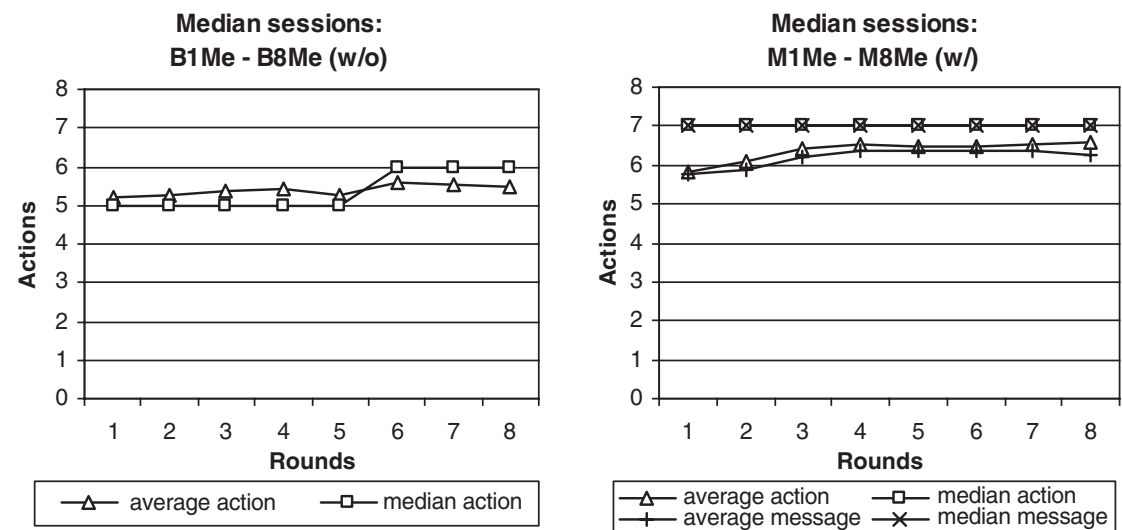

$\longrightarrow \Delta$ average action $\rightarrow \square-$ median action
+ average message $\rightarrow \leftarrow$ median message

Fig. 1. Comparison of baseline vs. pre-play Median sessions-all data.
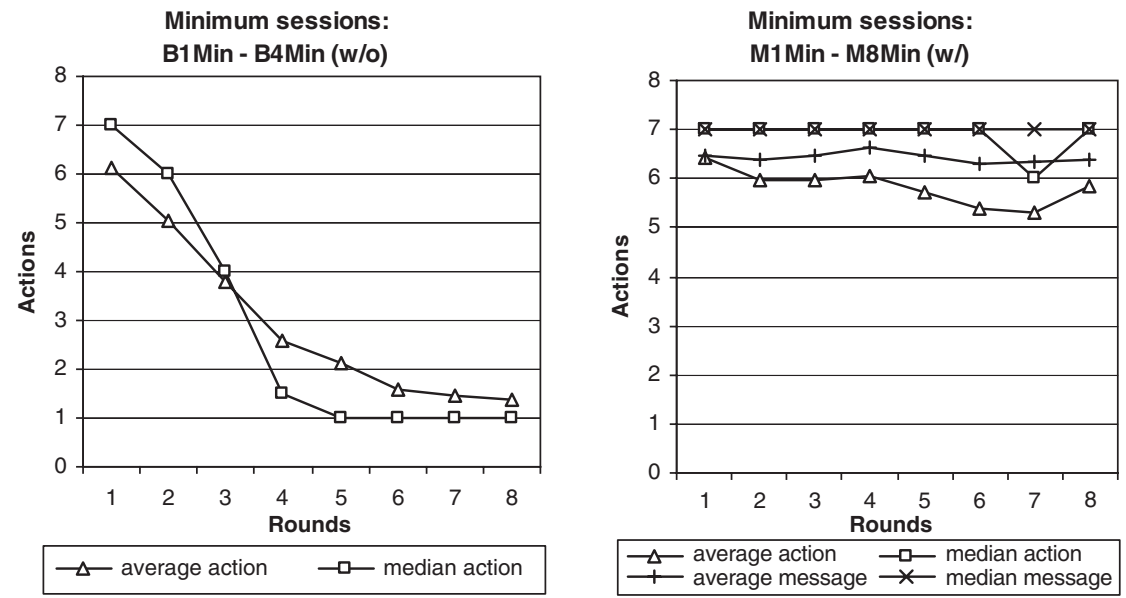

Fig. 2. Comparison of baseline vs. pre-play Minimum sessions-all data.

4,5 , or 6); median choices set a strong precedent for the following rounds. Slightly less than 30 percent of our subjects chose the action associated with the Pareto-efficient equilibrium in the first round. In contrast, in the baseline Minimum sessions about 80 percent of our subjects chose the action associated with the Pareto-efficient equilibrium in the first round. In three of the four sessions, however, one of the participants chose the action inducing the worst of the Pareto-ranked equilibria, setting in motion a swift change in the distribution of actions in the following round(s). In the communication sessions overall we see a higher incidence of efficient play in both the Median and Minimum games, as shown in the right panels of Figs. 1 and 2. This is further confirmed by the average payoffs per sessions that are reported in Tables 4 and 5. 
Table 4

Participants' earnings in Median sessions ${ }^{\mathrm{a}}$

\begin{tabular}{lllllllll}
\hline Session & B1Me & B2Me & B3Me & B4Me & B5Me & B6Me & B7Me & B8Me \\
\hline Earnings table & ETMe & ETMe & ETMe & ETMe & ETMe & ETMe & ETMe* & ETMe* \\
Avg. earnings & 10.04 & 8.65 & 10.21 & 9.28 & 7.78 & 9.06 & 7.57 & 7.49 \\
Max. earnings & 10.40 & 8.75 & 10.40 & 9.50 & 8.00 & 9.40 & 8.00 & 7.95 \\
Min. earnings & 8.90 & 8.45 & 9.50 & 8.90 & 7.15 & 8.10 & 6.65 & 6.60 \\
Session & M1Me & M2Me & M3Me & M4Me & M5Me & M6Me & M7Me & M8Me \\
Earnings table & ETMe & ETMe & ETMe & ETMe & ETMe & ETMe & ETMe* & ETMe* \\
Avg. earnings & 10.40 & 10.39 & 10.01 & 9.36 & 10.19 & 9.89 & 10.23 & 7.49 \\
Max. earnings & 10.40 & 10.40 & 10.40 & 9.80 & 10.40 & 10.40 & 10.40 & 8.05 \\
Min. earnings & 10.40 & 10.35 & 8.70 & 7.00 & 9.30 & 8.20 & 9.15 & 6.05
\end{tabular}

${ }^{\text {a }}$ ETMe or ETMe* are identical earnings tables, except that ETMe has the Pareto-dominant equilibrium in the upper left corner while ETMe* has it in the lower right. For details see footnote 6.

Table 5

Participants' earnings in Minimum sessions

\begin{tabular}{lllllllll}
\hline Session & B1Min & B2Min & B3Min & B4Min & & & \\
\hline Earnings table & ETMin & ETMin & ETMin & ETMin & & & & \\
Avg. earnings & 4.90 & 4.24 & 4.37 & 4.23 & & & & \\
Max. earnings & 6.10 & 5.50 & 5.20 & 5.60 & & & & \\
Min. earnings & 3.20 & 3.20 & 3.80 & 3.10 & & & & \\
Session & M1Min & M2Min & M3Min & M4Min & M5Min & M6Min & M7Min & Min \\
Earnings table & ETMin & ETMin & ETMin & ETMin & ETMin & ETMin & ETMin & ETMin \\
Avg. earnings & 8.81 & 9.48 & 7.26 & 4.72 & 7.79 & 8.46 & 3.90 & 10.40 \\
Max. earnings & 9.40 & 9.90 & 7.70 & 5.70 & 8.40 & 8.90 & 6.00 & 10.40 \\
Min. earnings & 8.40 & 9.40 & 6.70 & 3.80 & 7.40 & 8.20 & 2.00 & 10.40 \\
\hline
\end{tabular}

This gives us our first key observation: With repeated play, costless messages preceding games with Pareto-ranked equilibria can facilitate participants' coordination on the Paretodominant equilibrium, even for more than 2 players, and for both the Median and the Minimum games. This lends some support to the two central conjectures regarding the role of pre-play communication.

Fig. 3 summarizes the aggregate first-round choice frequencies of actions and messages for the Median game (left panel) and the Minimum game (right panel).

In the first round of the Median sessions with communication, more than 50 percent of our subjects ( 54.2 percent) chose the action associated with the Pareto-efficient equilibrium. This is up from about 29.2 percent in the corresponding baseline sessions, and a KolmogorovSmirnov (K-S) test allows us to reject the null hypothesis of equal distributions of action choices in the two Median game treatments with and without communication at the 5 percent confidence level (it does not reject the equality of distributions of action choices and messages in the pre-play treatment at the 5 percent level). Interestingly, again about 80 percent of our subjects chose the action associated with the Pareto-efficient equilibrium in the first round of the Minimum sessions with communication. K-S tests do not allow us to reject the null hypothesis of equal distributions of action choices in the baseline and the communication treatment, nor the null hypothesis of equal distributions of action 

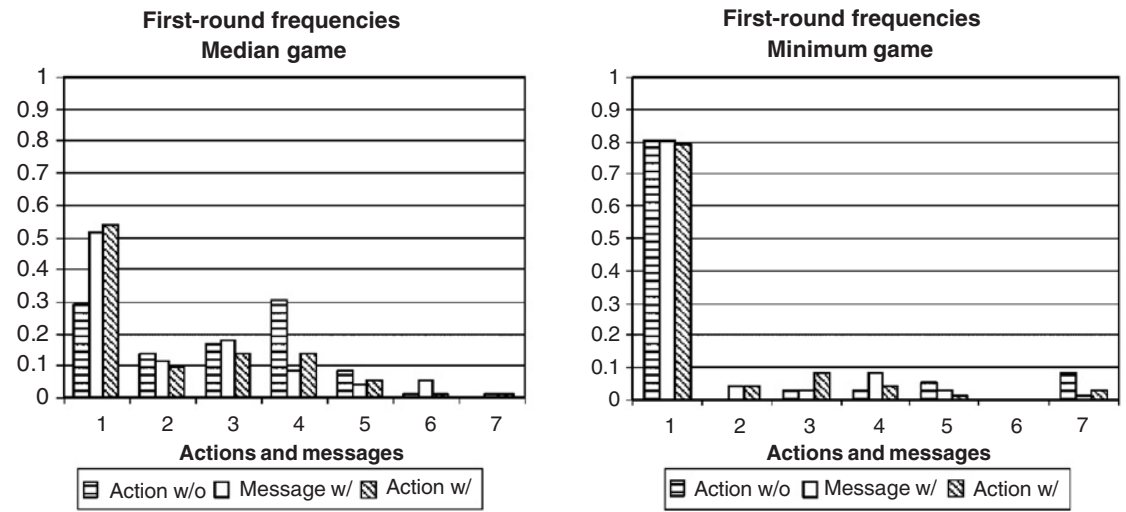

Fig. 3. First-round choices—distribution of actions and messages.

Table 6

Summary of convergence ${ }^{\mathrm{a}}$ results in Median sessions

\begin{tabular}{llr}
\hline & Convergence & Not \\
\hline B1Me-B8Me & 2 & 6 \\
M1Me-M8Me & 7 & 1 \\
\hline
\end{tabular}

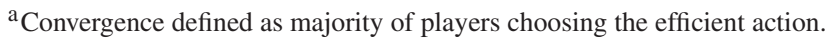

choices in the baseline treatment and messages in the pre-play treatment, nor the equality of distributions of action choices and messages in the pre-play treatment at the 5 percent level. We therefore have our second key observation: The first-round effect of pre-play communication differs across the games studied here. While there is a significant increase in the incidence of efficient play in the first round of the Median game, there is no such increase in the Minimum game. A likely explanation for this difference is that, given the relatively low frequency of efficient play in the first round of the Median game without communication, there is more scope for pre-play communication to make a difference. The fact that pre-play communication does not uniformly lead to efficiency or equilibrium in the first round in either the Median or the Minimum game qualifies our statement that our data support the two central conjectures regarding the effects of pre-play communication. Part of an explanation may be that some players remain attracted by safe actions in the Minimum game and may even use messages to secure payoffs in the Median game (see [5]).

In the terminal rounds of both communication treatments the frequency of efficient play is higher than in the treatments without communication. If we define "convergence" to efficient play as at least five players (i.e., the majority) choosing the efficient action, then "convergence" is achieved in the majority of communication sessions, and only in two Median game sessions without communication. Tables 6 and 7 summarize the convergence results.

A Fisher's exact test [22],[32, pp. 155-156] determines the convergence results for the two treatments with and without communication to be significantly different at the 5 percent 
Table 7

Summary of convergence ${ }^{\mathrm{a}}$ results in Minimum sessions

\begin{tabular}{lll}
\hline & Convergence & Not \\
\hline B1Min-B4Min & 0 & 4 \\
M1Min-M8Min & 6 & 2 \\
\hline
\end{tabular}

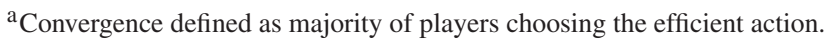

level ( $p=0.041$ ) for the Median sessions in Table 6 , and at the 10 percent level ( $p=0.061)$ for the Minimum sessions in Table 7. If we had run eight baseline sessions for the Minimum game, and if the additional sessions had produced the same result and hence the standard result in the literature, the difference would have been significant at the 1 percent level $(p=0.007)$. One of the two Minimum sessions with communication that did not converge to efficient play unraveled to the worst of the Pareto-ranked outcomes, while the other stabilized in the mid-range (with 8 of the 9 choices being labeled 4 and 5). We thus have our third key observation: While the terminal effect of pre-play communication is to increase efficient play in both games, this effect differs across the two games: It is more pronounced in the Minimum game, even though there two communication sessions unravel. The fact that in our experiments the full benefit of communication appears only with repeated interactions is perhaps indicative of messages serving as negotiation statements. Here the negotiation would be not so much over conflicting interests as over conflicting goals resulting from conflicting expectations, e.g., some players aiming for efficiency and others for making in the Median game a less than efficient median more certain. The unraveling in two instances of the Minimum game suggests that such negotiation need not necessarily lead to efficiency. It appears that repeated multi-sided communication may fail with risk in the underlying game and (or) absence of self-signaling messages. The completeness of unraveling in one of the two cases suggests that message meanings can indeed deteriorate to the point where they fail to serve as useful negotiation statements.

Our fourth key observation is that the dynamics of actions differ across the two communication games, both in the time paths of averages and the time paths of standard deviations. In either game we see non-degenerate action profiles throughout (see Figs. 1, 2, and 4). The average action increases in the Median game initially and then stays approximately constant (with the average reflecting some subjects "fooling around"), whereas it falls slightly in the Minimum game (only to recover somewhat in the last round). The average of the standard deviations of actions per session, which falls in both games, is much lower in the Minimum game communication treatment (see Fig. 4).

Lastly, as we observed before, unlike in the Median game, in the Minimum game we observe a bifurcation of behavior, with six sessions converging to the efficient action whereas the remaining two sessions unravel. Pre-play communication on average appears to significantly reduce strategic uncertainty and to concentrate beliefs near the efficient equilibrium in the early rounds of the Minimum game. In some instances however, this effect is overwhelmed by a few initial deviations from efficient play, which over time induce an increasing focus on the safe action in the course of repeated play.

In both games overall we see a close alignment of messages and actions, i.e. for the most part participants are "truthful" and take the action that matches their announcement. For 


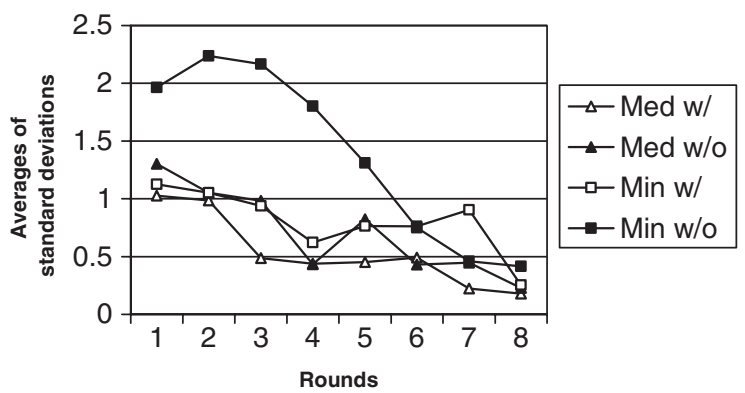

Fig. 4. Averages of standard deviations of actions. Standard deviations were calculated for each session and then averaged for each treatment.

example, in the first round of the Median experiment, 81 percent of those who announced they would choose the efficient action did so. In the Minimum experiment the corresponding percentage was even higher $(86.2 \%)$. The top and middle panel of Fig. 5 show that this pattern is maintained when we aggregate over all rounds for the Median game and the six efficient sessions of the Minimum game. In the two unraveling sessions of the Minimum game, we see two interesting departures from this pattern (see the bottom panel of Fig. 5).

First, a considerable number of players announce the efficient action but instead take a lower action. Second, a non-negligible fraction of players both announce and then take the safe action. This gives us our fifth key observation: In both games the majority of messages are both efficient and truthful, with departures from this pattern in the unraveling sessions of the Minimum game toward either overstatements of planned actions or to announcements of the safe action. The tendency toward truthfulness would be consistent with messages being widely perceived as self-committing and in most cases in the Minimum game this self-commitment property is dominating concerns about messages not being self-signaling. At the same time, the departures from truthfulness in the unraveling sessions are consistent with self-signaling and risk concerns exerting some influence on play. Announcements of the safe action, and then play of the safe action, do not fit neatly into the theory we have discussed. One plausible explanation might be that agents have other-regarding preferences: A player who intends to play the safe action would only harm other players by misleading them about his/her intentions.

While departures from truthfulness in the Minimum game tend to be overstatements of the planned action, there appears to be a slight tendency of the reverse phenomenon in the Median game. As shown in the right panel of Fig. 1, in all rounds the average action in the Median game with messages exceeds the average message ever so slightly (typically between one tenth and two tenths). One way to try to rationalize this observation is to recall that in the Median game there are multiple uses for communication: to enhance efficiency and to secure an expected median. Players who initially send messages to secure an inefficient expected median may reassess their expectations subsequently, in view of the messages they observe, and raise their action relatively to the message they sent. Note that in the auction experiments of VHBB [35], the observed median also tends to exceed the "message", i.e., the payoff associated with the observed median tends 


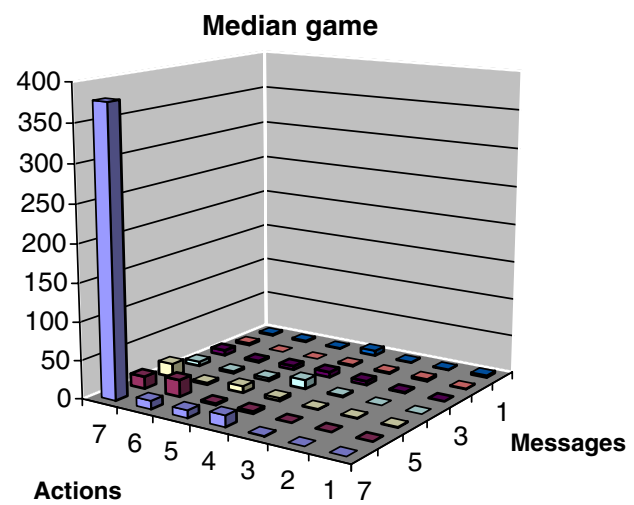

Minimum game, convergent

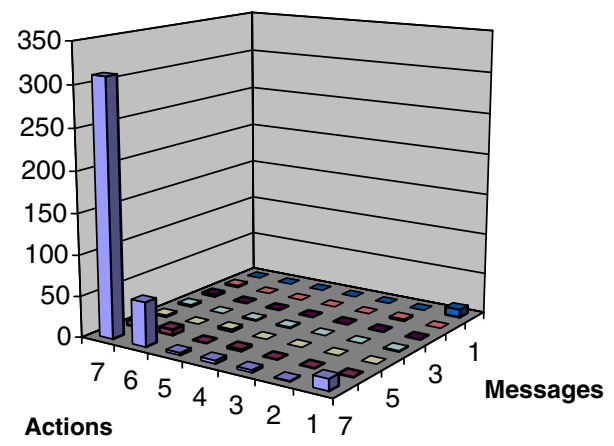

Minimum game, non-convergent

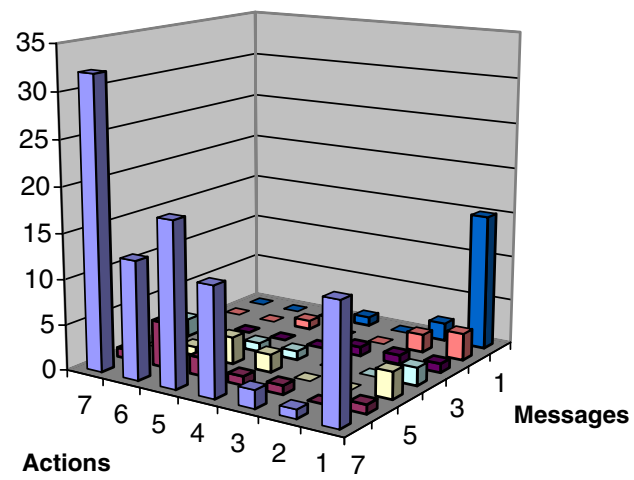

Fig. 5. Alignment of messages and actions. Top: Pre-play Median treatment across all rounds and all sessions. Middle: Pre-play Minimum treatment across all rounds in convergent sessions. Bottom: Pre-play Minimum treatment across all rounds in non-convergent sessions.

to exceed the price reached in the auction before convergence. In their case, the explanation is that nearly all participants in their experiments avoid the use of dominated actions. 


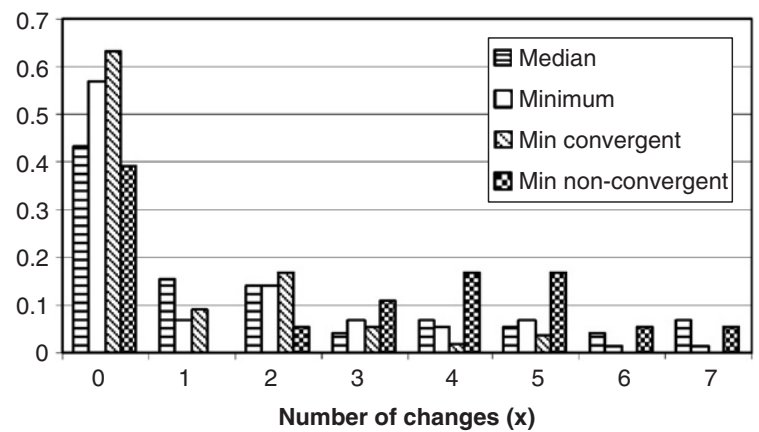

Fig. 6. Frequency of the number of times $(x)$ that players changed their message.

Our sixth key observation is that in both communication treatments a considerable number of players change their message at least once. This is illustrated in Fig. 6, which reports the frequency of the number of times that players changed their messages for both communication treatments, and separately for the convergent and non-convergent sessions of the pre-play Minimum treatment.

Averaged across the communication treatments, 50 percent of the players changed their message at least once.

On average the rate of change gradually declines over time in both treatments, from more than 30 percent of players changing their message between the first and second round to about 15 percent of players changing their message between the last two rounds. Once again, the two non-convergent sessions of the Minimum game are exceptional, with the rate of change being above 30 percent between any two consecutive rounds but the first two. The fact that a substantial number of players keep changing their messages has at least some of the flavor of the secret handshake argument. A player who changes his message induces a new message profile and might hope that the new profile is more likely to induce efficient play.

\section{Conclusion}

Our central result is that with repeated interaction costless messages preceding games with Pareto-ranked equilibria can dramatically facilitate participants' coordination on the Pareto-dominant equilibrium, even for more than 2 players. Costless messages thus help overcome well-documented problems of strategic uncertainty, equilibrium selection, and coordination failure. These overall effects of pre-play communication in our data seem robust to the parameterization of the payoff matrices and the order statistics chosen. There are differences in the role that pre-play communication plays over time in these games, with the salutary effect being initially stronger in the median game but eventually being more pronounced in the minimum game.

Regarding theories that have been advanced for signaling intentions in games, we find that players appear to rely on self-commitment properties of messages. We find that neither 
risk in the underlying game nor the absence of self-signaling messages entirely undermines the effectiveness of pre-play messages, while both help to account for those instances in which we observe unraveling and divergence of messages and actions. We observe that the full benefit of adding communication is only realized with repeated interactions, which may indicate the use of messages as negotiation statements. At the same time we find that a nonnegligible number of subjects do change their messages over time, possibly hoping to affect others' action choices, which is reminiscent of the use of messages as secret handshakes.

Our work suggests a number of extensions, for example, dealing with alternative communication protocols, the incentive structure in the underlying game, timing issues, methods for collecting information about players' strategies, and the richness of the language available to players. A natural alternative communication protocol would permit repeated communication before actions are taken, as in Farrell [20], Rabin [30] and Jamison [24]. Perhaps in conjunction with altering the incentive structure to induce conflict over equilibria, this would help us to better understand the role of messages as negotiation statements. Regarding timing, it is natural to ask whether communication can be effective if one or more rounds of play have already preceded it. For example, is there still a role for communication after a few rounds of play of the minimum game without communication? If instead of collecting only information about players' messages and actions, we asked for their strategies in the communication game, we would be able to assess the role of secret handshakes more directly. Finally, it is conceivable that giving players access to a richer language, or free-form communication, would affect coordination.

\section{Acknowledgments}

Financial support by the National Science Foundation under award numbers SBR9410588 and SBR-9808947 (Blume) and SBR-95421007 (Ortmann) is gratefully acknowledged. We thank Vince Crawford, Ernst Fehr, John Fitzgerald, Oliver Kirchkamp, Jean-Francois Mertens, an anonymous referee for, and the associate editor of, this journal for their constructive comments, Steve Deitz, Anthony Roy, and Charles Kannair for their help in programming and running the experiments, and Michal Ostatnicky for his help in the data analysis.

\section{References}

[1] R. Aumann, Nash equilibria are not self-enforcing, in: J.J. Gabszewicz, J.-F. Richard, L.A. Wolsey (Eds.), Economic Decision Making: Games, Econometrics and Optimization, Elsevier, North-Holland, Amsterdam, 1990, pp. 201-206.

[2] K. Basu, J.W. Weibull, Strategy subsets closed under rational behavior, Econ. Letters 36 (1991) 141-146.

[3] S.K. Berninghaus, K.M. Ehrhart, Time horizon and equilibrium selection in tacit coordination games: experimental results, J. Econ. Behav. Organ. 37 (1998) 231-248.

[4] A. Blume, Communication, risk, and efficiency in games, Games Econ. Behav. 22 (1998) 171-202.

[5] A. Burton, M. Sefton, Risk, pre-play communication and equilibrium, Games Econ. Behav. 46 (2004) 23-40.

[6] G.P. Cachon, C.F. Camerer, Loss-avoidance and forward induction in experimental coordination games, Quart. J. Econ. 111 (1996) 165-194. 
[7] C.F. Camerer, Behavioral Game Theory, Princeton University Press, Princeton, 2003.

[8] G. Charness, Self-serving cheap talk: a test of Aumann's conjecture, Games Econ. Behav. 33 (2000) 177-194.

[9] K. Clark, S. Kay, M. Sefton, When are Nash equilibria self-enforcing? An experimental analysis, Int. J. Game Theory 29 (2001) 435-456.

[10] R.C. Cooper, D. De Jong, R. Forsythe, T. Ross, Communication in coordination games, Quart. J. Econ. 107 (1992) 739-771.

[11] V. Crawford, An evolutionary interpretation of Van Huyck, Battalio, and Beil's experimental results on coordination, Games Econ. Behav. 3 (1991) 25-59.

[12] V. Crawford, Adaptive dynamics in coordination games, Econometrica 63 (1995) 103-143.

[13] V. Crawford, Theory and experiment in the analysis of strategic interaction, in: D.M. Kreps, K.F. Wallis (Eds.), Advances in Economics and Econometrics: Theory and Applications, Seventh World Congress, vol. 1, Cambridge University Press, Cambridge, 1997, pp. 206-242.

[14] V. Crawford, A survey of experiments on communication via cheap talk, J. Econ. Theory 78 (1998) $286-298$.

[15] V. Crawford, Introduction to experimental game theory, J. Econ. Theory 104 (2002) 1-15.

[16] V. Crawford, B. Broseta, What price coordination? The efficiency-enhancing effect of auctioning the right to play, Amer. Econ. Rev. 88 (1998) 198-225.

[17] D.D. Davis, C.A. Holt, Experimental Economics, Princeton University Press, Princeton, 1993.

[18] G. Devetag, Coordination and information in critical mass games: an experimental study, Exper. Econ. 6 (2003) 53-73.

[19] J. Duffy, N. Feltovich, Do actions speak louder than words? An experimental comparison of observation and cheap talk, Games Econ. Behav. 39 (2002) 1-27.

[20] J. Farrell, Cheap talk, coordination and entry, RAND J. Econ. 18 (1987) 34-39.

[21] J. Farrell, M. Rabin, Cheap talk, J. Econ. Perspect. 10 (1996) 103-118.

[22] R.A. Fisher, The logic of inductive inference, J. Roy. Statistical Society 98 (1935) 39-54.

[23] S. Hurkens, Multi-sided pre-play communication by burning money, J. Econ. Theory 69 (1996) $186-197$.

[24] J. Jamison, Valuable cheap talk and equilibrium selection, Northwestern University Working Paper, 2002.

[25] Y.-G. Kim, J. Sobel, An evolutionary approach to pre-play communication, Econometrica 63 (1995) 1181-1193.

[26] D.M. Kreps, A Course in Microeconomic Theory, Princeton University Press, Princeton, 1995.

[27] R.D. Luce, H. Raiffa, Games and Decisions, Wiley, New York, 1957.

[28] A. Matsui, Cheap-talk and cooperation in society, J. Econ. Theory 54 (1991) 245-258.

[29] H. Moulin, Game Theory for the Social Sciences, New York University Press, New York, 1986.

[30] M. Rabin, A model of pre-game communication, J. Econ. Theory 63 (1994) 370-391.

[31] A.J. Robson, Efficiency in evolutionary games: Darwin, Nash and the secret handshake, J. Theoret. Biol. 144 (1990) 379-396.

[32] K.J. Rothman, Modern Epidemiology, Little, Brown and Company, Boston, 1986.

[33] J.B. Van Huyck, R.C. Battalio, R.O. Beil, Tacit coordination games, strategic uncertainty, and coordination failure, Amer. Econ. Rev. 80 (1990) 234-248.

[34] J.B. Van Huyck, R.C. Battalio, R.O. Beil, Strategic uncertainty, equilibrium selection, and coordination failure in average opinion games, Quart. J. Econ. 106 (1991) 885-911.

[35] J.B. Van Huyck, R.C. Battalio, R.O. Beil, Asset markets as an equilibrium selection mechanism: coordination failure, game form auctions, and tacit communication, Games Econ. Behav. 5 (1993) 485-504.

[36] J.B. Van Huyck, R.C. Battalio, F.W. Rankin, Evidence on learning in coordination games, Texas A\&M University Working Paper, 2001.

[37] K. Waerneryd, Evolutionary stability in unanimity games with cheap-talk, Econ. Letters 36 (1991) 375-378. 